\title{
Iris Recognition System Using Support Vector Machines
}

\author{
Hasimah and Momoh J. E. Salami \\ University Malaysia Perlis (UniMAP), International Islamic University Malaysia (IIUM) \\ Malaysia
}

\section{Introduction}

In the modern world, a reliable personal identification infrastructure is required to control the access in order to secure areas or materials. Conventional methods of recognizing the identity of a person by using passwords or cards are not altogether reliable, because they can be forgotten, stolen, disclosable, or transferable (Zhang, 2000). Biometric technology, which is based on physical and behavioral features of human body such as face, fingerprint, hand shapes, iris, palmprint, keystroke, signature and voice, (Lim et al., 2001, Zhang, 2000, Zhu et al., 1999) has now been considered as an alternative to existing systems in a great deal of application domains such as bank Automatic Teller Machines (ATM), telecommunication, internet security and airport security.

Each biometric technology has its own advantages and disadvantages based on their usability and security. Among the various traits, iris recognition has attracted a lot of attention. Iris is an internal (yet externally visible) organ of the eye, which is well protected from the environment and its patterns are apparently stable throughout the life. The iris consists of variable sized hole called pupil. The average diameter of the iris is $12 \mathrm{~mm}$, and the pupil size can vary from $10 \%$ to $80 \%$ of the iris diameter. It has the great mathematical advantage that its pattern variability amongst people is enormous (Daugman, 2002).

The number of features in human iris is large. Its complex pattern can contain many distinctive features such as arching, ligaments, furrows, ridges, crypts, rings, corona, freckles and zigzag collarette (Wildes, 1999, Daugman, 2002) for personal identification. Fig. 1 is an example of human iris. That is because every iris has fine unique texture and does not change over time. In addition, iris pattern can have up to 249 independent degrees of freedom. Because of high randomness in the iris pattern, it has made the technique more robust and it is very difficult to deceive an iris pattern (Daugman, 2003). Unlike other biometric traits, iris recognition is the most accurate and non-invasive biometric for secure authentication and positive identification. This proposed system use a publicly availably library for iris recognition written in MATLAB (Masek, 2003).

Due to the advantages of iris recognition systems which offer reliable and effective security in the present day, this research proposed the use of iris-based as verification system system to identify the person's identity. This research work adopts Support Vector Machines (SVMs) as pattern classification techniques which are based on iris code model which the feature vector size is transformed to one-dimension vector which reduces to $1 \times 480$ by using averaging techniques (each segment is divided by 20) contains the average value to 
recognize an authorized user and unauthorized user. The effectiveness of the proposed system is evaluated based on False Rejection Rate (FRR) and False Acceptance Rate (FAR).

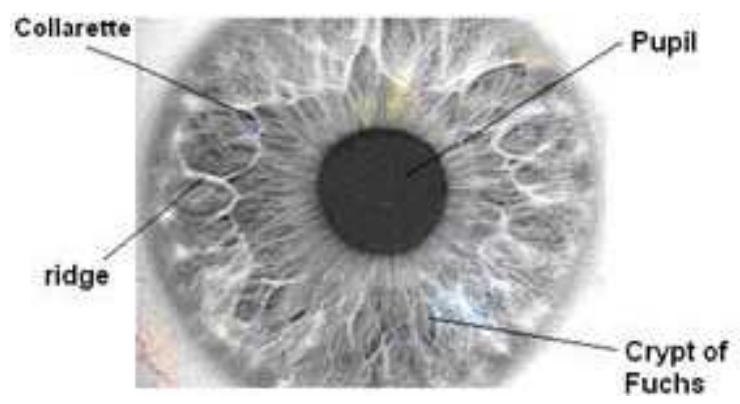

Fig. 1. An iris which has highly complex unique texture.

\section{Iris image acquisition}

Image acquisition is meant to produce image of user's eye region. A high-quality image of the iris has to be captured in order for the iris recognition system to work efficiently. The acquired image of the iris must have sufficient resolution and sharpness to support recognition. Besides, it is important to have good contrast in the interior iris pattern. The brightness of illumination which is not uniformly distributed will result in poor quality images with lots of spatial reflections. Images captured using infrared camera has good quality with high contrast and low reflections (Wildes, 1999).

\section{Iris image preprocessing}

The acquired image that contains irrelevant parts (e. eyelid, eyelash, pupil, etc) should be removed. For the purpose of analysis, the original image needs to be preprocessed. The preprocessing is composed of two steps: iris localization/ segmentation and normalization.

\subsection{Iris localization / segmentation}

The first stage of iris recognition system is to isolate the actual iris region in a digital eye. The purpose of iris localization is to localize an acquired image that corresponds to an iris. The iris region, shown in Fig. 2, can be approximated by two circles, one for the iris/sclera boundary and the other is interior to the first, for the iris/pupil boundary (Daugman, 2002). The eyelids and eyelashes are normally occluding the upper and lower parts of the iris region. Eyelids and eyelashes are isolated from the detected iris image by considering them as noise because they degrade the performance of the system.

Daugman (2002) proposed integro-differential operator to detect the centre and diameter of the iris and used the differential operators to detect the pupil. That is,

$$
\max _{\left(r, x_{p}, y_{o}\right)}\left|G_{\sigma}(r) * \frac{\partial}{\partial r} \oint_{r, x_{o}, y_{o}} \frac{I(x, y)}{2 \Pi r} d s\right|
$$

where $I(x, y)$ is the eye image, $r$ is the radius to search for, $G_{\sigma}(r)$ is a Gaussian smoothing function, $s$ is the contour of the circle given by $r, x_{0}, y_{0}$ (Masek, 2003). Wildes (1999) used 

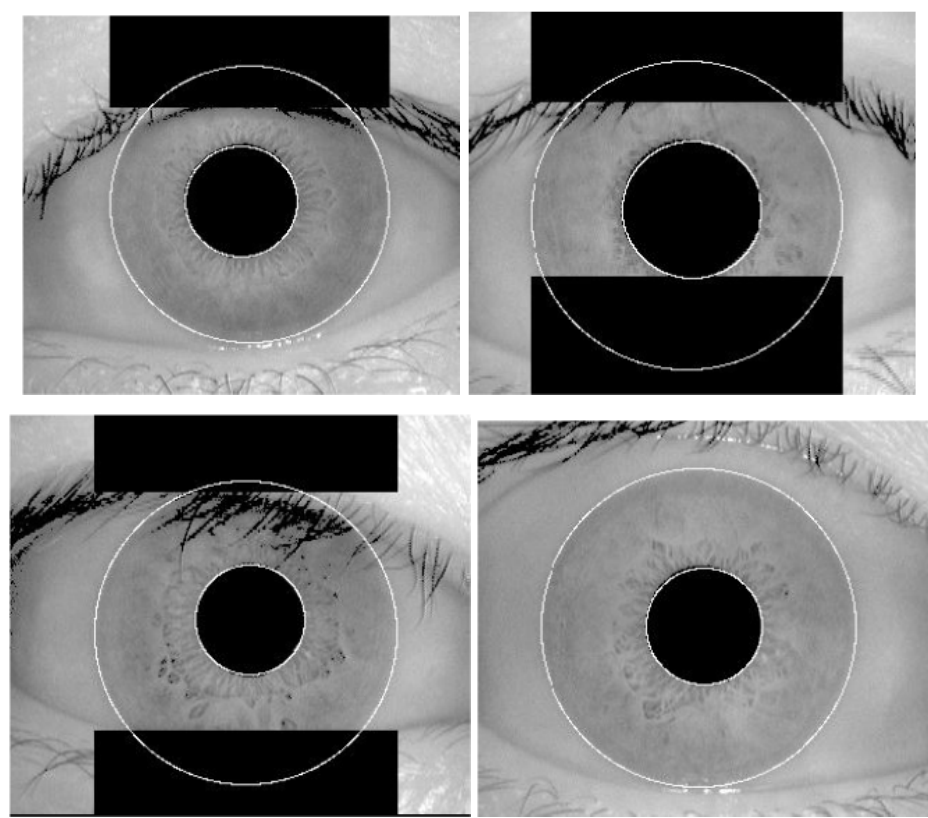

Fig. 2. Automatic segmentation of various images from the CASIA database. Black regions denote detected eyelid and eyelash regions (Masek, 2003).

the first derivative of image intensity to find the location of edges corresponding to the borders of the iris. This approach explicitly models the upper and lower eyelids with parabolic arcs whereas Daugman (2002) excludes the upper and lower portion of the image in its modal.

\subsection{Iris normalization}

The localized iris part is transformed into polar coordinates system so that it has fixed dimensions and also to overcome imaging inconsistencies. The annular iris region is transformed into rectangular region where the iris texture is analyzed. The Cartesian to polar transform of the iris region is based on the Daugman's Rubber Sheet model as shown in Fig. 3. The rubber sheet model takes into account pupil dilation and size dimensions. Therefore, the iris region is modeled as a flexible rubber sheet anchored at the boundary with the pupils centre as the reference point (Masek, 2003). Daugman remap each point within the iris region to a pair of polar coordinates $(r, \theta)$ where $r$ is on the interval $[0,1]$ and $\theta$ is angle $[0,2 \Pi]$. The remapping of the iris region is modeled as,

$$
\mathrm{I}(\mathrm{x}(\mathrm{r}, \theta), \mathrm{y}(\mathrm{r}, \theta)) \rightarrow \mathrm{I}(\mathrm{r}, \theta)
$$

with

$$
\begin{aligned}
& x(r, \theta)=(1-r) x_{p}\left(\theta+r x_{1}(\theta)\right. \\
& y(r, \theta)=(1-r) y_{p}\left(\theta+\operatorname{ry}_{1}(\theta)\right.
\end{aligned}
$$


where $I(x, y)$ is the iris region image, $(x, y)$ are the original Cartesian coordinates, $(r, \theta)$ are corresponding normalized polar coordinates, and $\mathrm{x}_{\mathrm{p}}, \mathrm{y}_{\mathrm{p}}$ and $\mathrm{x}_{\mathrm{l}}, \mathrm{y}_{\mathrm{l}}$ are the coordinates of the pupil and iris boundaries along the $\theta$ direction.
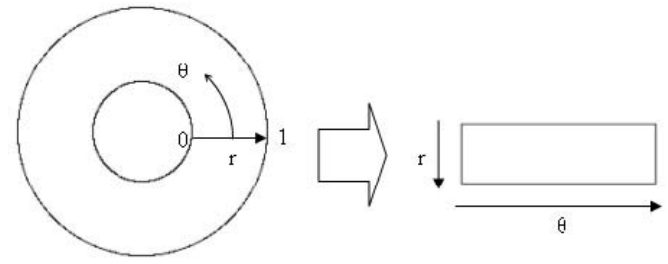

Fig. 3. Daugman's Rubber Sheet Model.

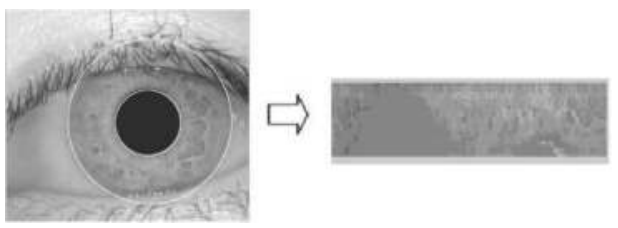

Fig. 4. Iris normalized into polar coordinates.

For normalization of iris regions, a technique based on Daugman's rubber sheet model was employed in the proposed system. The centre of pupil was considered as the reference point and radial vectors pass through the iris region (Masek, 2003).

\section{Feature extraction / Encoding}

The iris has a particularly interesting structure and provides abundant texture information. In order to recognize the individuals accurately, the most discriminating features that present in the region must be extracted. Only the significant features of the iris must be encoded. There are many algorithm that are available for feature extraction such as wavelet encoding, Gabor filters (Daugman, 2002) , Log-Gabor filter (Field, 1987), zero-crossings of the 1D wavelet (Boles et. al, 1998), Haar wavelet (Lim et. al, 2001) and Laplacian of Gaussian filters (Widles, 1996).

In the proposed system, Masek's algorithm was used for feature encoding by convolving the normalized iris pattern with 1D Log-Gabor wavelets. Log-Gabor filters are constructed using,

$$
G(f)=\exp \left(\frac{-\left(\log \left(f / f_{0}\right)\right)^{2}}{2\left(\log \left(\sigma / f_{0}\right)\right)^{2}}\right)
$$

where fo represents the centre frequency , $\sigma$ gives bandwidth of the filter. The 2D normalized pattern is broken up into a number of 1D signal, and then these signal are convolved with 1D Gabor wavelet. The rows of the 2D normalized pattern are taken as the 1D signal. Each row corresponds to a circular ring on the iris region. The phase information in the pattern only is used because the phase angles are assigned regardless of the image contrast. Extraction of the phase information, according to Daugman, is done using 2D Gabor wavelets. It determines which quadrant the resulting phasor lays using the wavelet, 


$$
h\{\operatorname{Re}, \operatorname{Im}\}=\operatorname{sgn}_{\{\operatorname{Re}, \operatorname{Im}\}} \iint_{\rho \phi} I(\rho, \phi) e^{-i \omega\left(\theta_{0}-\phi\right)} e^{-\left(r_{0}-\rho\right)^{2} / \alpha^{2}} e^{-\left(\theta_{0}-\phi\right)^{2} / \beta^{2}} \rho d \rho d \phi
$$

where, $\mathrm{h}\{\operatorname{Re}, \operatorname{Im}\}$ has the real boundary and imaginary part, each having the value 1 or 0 , depending on which quadrant it lies in (Daugman, 2002). $I(\rho, \phi)$ is the raw iris image in a dimensionless polar coordinate system that is size and translation-invariant, and which also corrects for pupil dilation; $a$ and $\beta$ are the multi-scale 2D wavelet size parameters, spanning an 8 -fold range from $0.15 \mathrm{~mm}$ to $1.2 \mathrm{~mm}$ on the iris; $\omega$ is wavelet frequency, spanning 3 octaves in inverse proportion to $\beta$; and $\left(r_{0}, \theta_{0}\right)$ represent the polar coordinates of each region of iris for which the phasor coordinates $h\left\{R_{e}, I_{m}\right\}$ are computed.

\section{Matching}

To verify a person's identity, the calculated iris template need to be matched with the stored template. Matching algorithm that normally used are Hamming Distance, Weighted Euclidean Distance and Normalized Correlation. In the proposed system, SVM is used as pattern matching method to verify a person's identity based on the iris code. The following section will describe the SVM used as pattern matching.

\subsection{Hamming distance (HD)}

The Hamming distance (HD) gives a measure of how many bits are the same between bit patterns. Using the Hamming distance of two bit patterns, a decision can be made as to whether the two patterns are generated from different irises or from the same ones.

The Hamming distance, $H D$, is defined as the sum of disagreeing bits (sum of the exclusiveOR between $X$ and $Y$ ) over $N$, the total number of bits in the bit pattern, that is,

$$
H D=\frac{1}{N} \sum_{j=1}^{N} X_{j}(X O R) Y_{j}
$$

\subsection{Weighted Euclidean Distance}

Zhu et al. (2000) employed Weighted Euclidian Distance (WED) for comparing two templates, especially if the template is composed of integer values. The weighting Euclidean distance gives a measure of how similar a collection of values are between two templates by using the following equation,

$$
W E D(k)=\sum_{i=1}^{N} \frac{\left(f_{i}-f_{i}^{(k)}\right)^{2}}{\left(\delta_{i}^{(k)}\right)^{2}}
$$

where $f_{i}$ is the $i^{\text {th }}$ feature of the unknown iris, and $f_{i}^{(k)}$ is the $i^{\text {th }}$ feature of iris template, $k$, and $\delta_{i}^{(k)}$ is the standard deviation of the $i^{\text {th }}$ feature in iris template $k$. The unknown iris template is found to match iris template $k_{o}$, when WED $\left(k_{0}\right)$ has a minimum value.

\subsection{Normalized Correlation}

The normalized correlation (NC) between the acquired and database representation has been reported by Wildes et al. to ascertain the accuracy of matching (Masek, 2003). This is expressed as, 


$$
N C=\frac{\sum_{i=1}^{n} \sum_{j=1}^{m}\left(p_{1}[i, j]-\mu_{1}\right)\left(p_{2}[i-j]-\mu_{2}\right)}{n m \sigma_{1} \sigma_{2}}
$$

where $p_{1}$ and $p_{2}$ are two images of size $n m, \mu_{1}$ and $\sigma_{1}$ are the mean and standard deviation of $p_{1}$, and $\mu_{2}$ and $\sigma_{2}$ are the mean and standard deviation of $p_{2}$.

\section{Iris-based verification system}

Iris recognition utilizes the distinctive features of human iris in order to identify or verify the identity of individuals. Traditionally, iris recognition system has been used in highsecurity physical access application, for example in ATMs and kiosks for banking and travel application. Iris recognition's strength includes the following:

1. It has the potential for exceptionally high levels of accuracy.

2. It is capable of providing reliable identification as well as verification.

3. It maintains stability of characteristic over a lifetime.

Fig. 5(a) and Fig. 5(b) depict the essential steps of the proposed iris verification system. The system has two sub-systems: the iris training or enrollment phase and iris testing (operational) phase. The training phase is shown in Fig. 5(a).

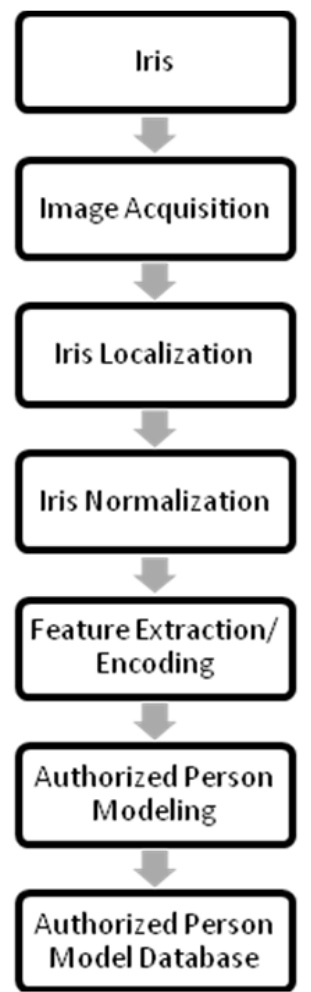

Fig. 5(a). Basic structure of iris-based verification system: Training phase. 
In this phase, the authorized persons registered their iris images. Fig. 5(b) shows the process involved in the second phase. Thus, a person who wants to access the system is required to enter the claimed identity and his/her iris image. Furthermore, the captured iris image is processed and compared with the claimed person model to verify his claim. The iris testing phase has a decision process in which the system decides whether the extracted features from the given iris image matches with the model of the claimed person.

In order to give access or reject, a threshold is set. If the degree of similarity between a given iris image and its model than a given threshold, then the user will access the system, otherwise the user is rejected. The system computes the following decisions: the authorized person is accepted, the authorized person is rejected, the unauthorized person (impostor) is accepted and the unauthorized person (impostor) is rejected.

Taking the advantages of iris recognition systems which offer reliable and effective security in the present day, the use of iris-based verification system to identify the person's identity is also examined in this research work. This research adopts Support Vector Machines (SVMs) as pattern classification techniques which are based on iris code model to recognize an authorized user. The effectiveness of the proposed system is evaluated based on False Rejection Rate (FRR) and False Acceptance Rate (FAR).

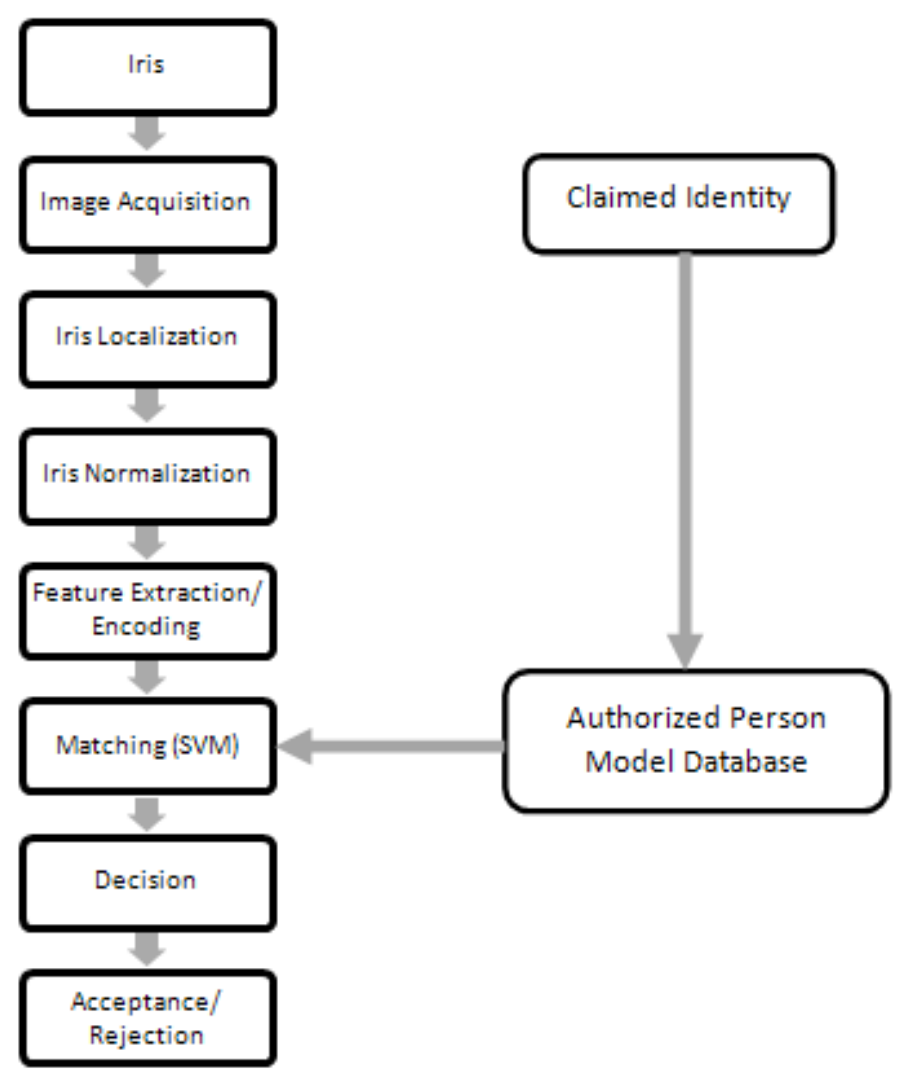

Fig. 5(b). Basic structure of iris-based verification system: Testing phase. 
Furthermore, this research aims to develop a system that would have both low FRR and FAR so as attain both high usability and high security.

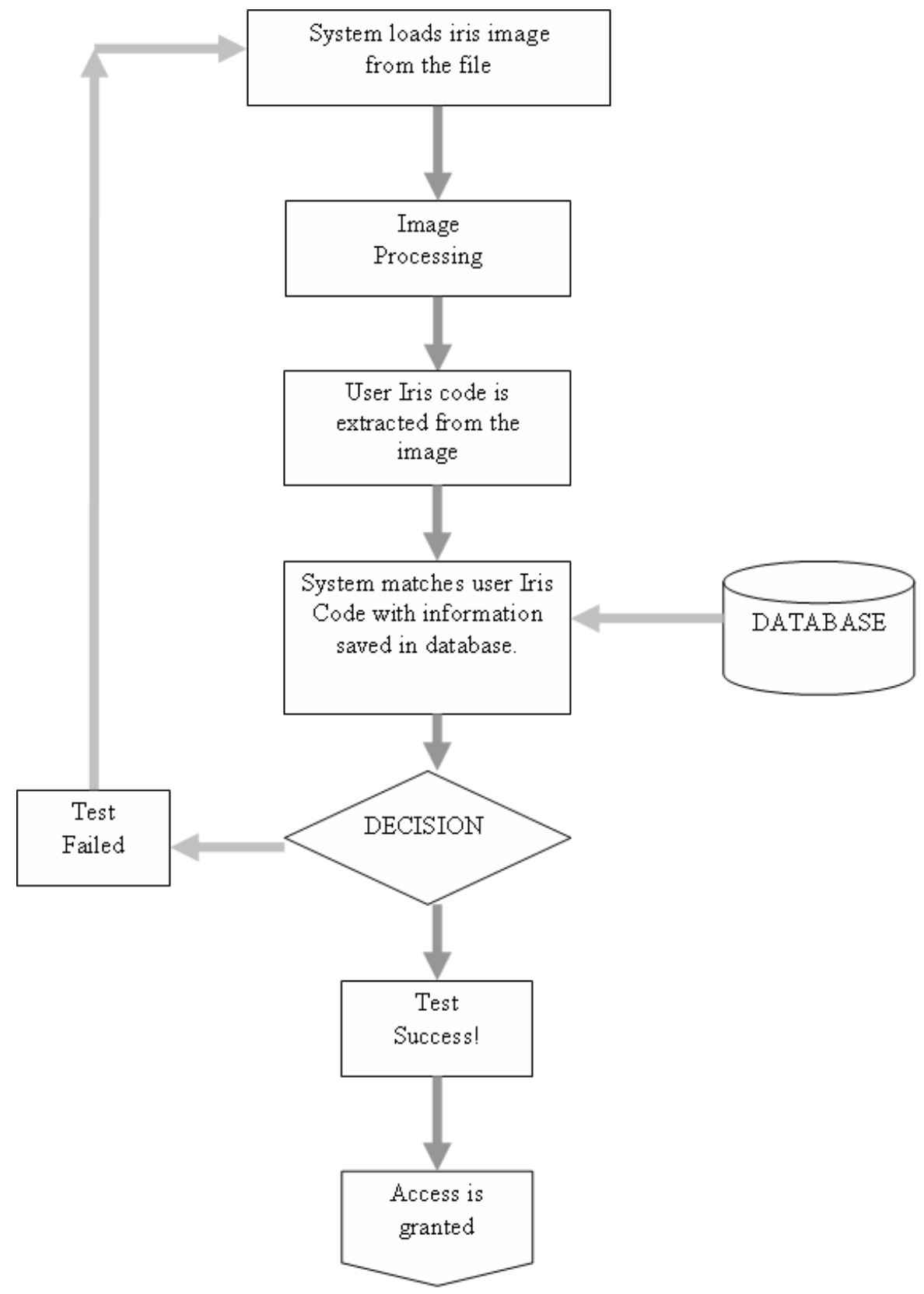

Fig. 6. Flowchart of iris-based authentication system. 


\section{Support vector machines}

SVM is a relatively new learning machine technique, which is based on the principle of structural risk minimization (minimizing classification error). A SVM is binary classifier that optimally separates the two classes of data (Burges, 1998). There are two important aspects in the development of SVM as classifier. The first aspect is determination of the optimal hyperplane which will optimally separate the two classes and the other aspect is transformation of non-linearly separable classification problem into linearly separable problem. This section will discuss in brief the two aspects of the SVM development. Detail discussion on the SVM can be found in the introductory text by (Burges, 1998), for more detail description, (Cristianini, 2000). Fig. 7 shows linearly separable binary classification problem with no possibility of miss-classification data. Let $\mathbf{x}$ and $\mathbf{y}$ be a set of input feature vector and the class label repectively. The pair of input feature vectors and the class label can be represented as tuples $\left\{x_{i}, y_{i}\right\}$ where $i=1,2, \ldots, N$ and $y= \pm 1$. In the case of linear separable problem, there exists a separating hyperplane which defines the boundary between class 1 (labeled as $\mathrm{y}=1$ ) and class 2 (labeled as $\mathrm{y}=-1$ ). The separating hyperplane is,

$$
\mathrm{w} \cdot \mathrm{x}+\mathrm{b}=0
$$

which implies

$$
\mathrm{y}_{\mathrm{i}}\left(\mathrm{w} \cdot \mathrm{x}_{\mathrm{i}}+\mathrm{b}\right) \geq 1, \mathrm{i}=1,2, \ldots \mathrm{N}
$$

Basically, there are numerous possible values of $\{\mathbf{w}, b\}$ that create separating hyperplane. In SVM only hyperplane that maximizes the margin between two sets is used. Margin is the distance between the closest data to the hyperlane.

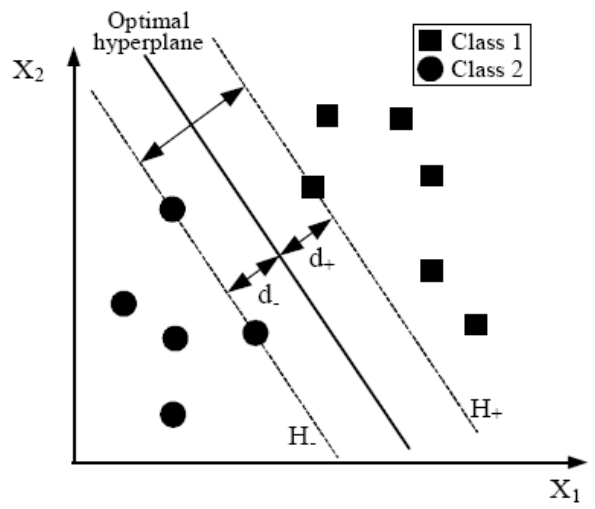

Fig. 7. SVM with Linear separable data.

Referring to Fig. 7 the margins are defined as $d+$ and $d-$. The margin will be maximized in the case $d_{+}=d_{-}$. Moreover, training data in the margins will lie on the hyperplanes $\mathrm{H}_{+}$and $\mathrm{H}$-. The distance between hyperplane $\mathrm{H}+$ and $\mathrm{H}$ - is,

$$
\mathrm{d}_{+}+\mathrm{d}_{-}=\frac{2}{\|\mathrm{w}\|}
$$


As $\mathrm{H}+$ and $\mathrm{H}$ - are the hyperplane in which the closest training data to the optimal hyperplane, then there is no training data which fall between $\mathrm{H}+$ and $\mathrm{H}-$. This means the hyperplane that separates optimally the training data is the hyperplane which minimizes $\|\mathrm{w}\|^{2}$ so that the distance of (12) is maximized. However, the minimization of $\|\mathrm{w}\|^{2}$ is constrained by (11). When the data is non-separable, slack variables, $\xi \mathrm{i}$, are introduced into the inequalities for relaxing them slightly so that some points allow to lie within the margin or even being misclassified completely. The resulting problem is then to minimize,

$$
\frac{1}{2}\|\mathrm{w}\|^{2}+\mathrm{C}\left(\sum_{\mathrm{i}} \mathrm{L}\left(\xi_{\mathrm{i}}\right)\right)
$$

where $C$ is the adjustable penalty term and $L$ is the loss function. The most common used loss function is linear loss function, $\mathrm{L}\left(\xi_{\mathrm{i}}\right)=\xi_{\mathrm{i}}$. The optimization of (13) with linear loss function using Lagrange multipliers approach is to maximize,

$$
L_{D}(w, b, a)=\sum_{i}^{N} a_{i}-\frac{1}{2} \sum_{i=1}^{N} \sum_{i=1}^{N} a_{i} a_{j} y_{i} y_{j}\left\langle x_{i} \cdot x_{j}\right\rangle
$$

subject to

$$
0 \leq a_{i} \leq C
$$

and

$$
\sum_{i}^{N} a_{i} y_{i}
$$

where $a_{i}$ is the Lagrange multipliers. This optimization problem can be solved by using standard quadratic programming technique. Once the problem is optimized, the parameters of optimal hyperplane are,

$$
\mathrm{w}=\sum_{\mathrm{i}}^{\mathrm{N}} \mathrm{a}_{\mathrm{i}} \mathrm{y}_{\mathrm{i}} \mathrm{x}_{\mathrm{i}}
$$

As matter of fact, $a_{i}$ is zero for every $x_{i}$ except the ones that lie on the margin. The training data with non-zero $a_{i}$ are called as support vectors. In the case of a non-linear separable problem, a kernel function is adopted to transform the feature space into higher dimensional feature space in which the problem become linearly separable. Typical kernel functions commonly used are listed in Table 1.

\begin{tabular}{|l|c|}
\hline Kernel & $\mathrm{K}\left(\mathrm{x}, \mathrm{x}_{\mathrm{i}}\right)$ \\
\hline Linear & $x^{T} \bullet x_{j}$ \\
\hline Polynomial & $\left(x^{T} \bullet x_{j}+1\right)^{d}$ \\
\hline Gaussian RBF & $\exp \left(\frac{-\left\|x-x_{j}\right\|^{2}}{2 \sigma^{2}}\right)$ \\
\hline
\end{tabular}

Table 1. Formulation for Kernel function. 


\section{Performance measures}

This proposed system in general makes four possible decisions; the authorized person is accepted, the authorized person is rejected, the unauthorized person (impostor) is accepted and the unauthorized person (impostor) is rejected. The accuracy of the proposed system is then specified based on the rate in which the system makes the decision to reject the authorized person and to accept the unauthorized person. False Rejection Rates (FRR) is used to measure the rate of the system to reject the authorized person and False Acceptance Rates (FAR) used to measure the rates of the system to accept the unauthorized person. Both performances are can be expressed as:

$$
\begin{aligned}
& \mathrm{FRR}=\frac{\mathrm{NFR}}{\mathrm{NAA}} \times 100 \% \\
& \mathrm{FAR}=\frac{\mathrm{NFA}}{\mathrm{NIA}} \times 100 \%
\end{aligned}
$$

NFR is referred to the numbers of false rejections and NFA is referred to the number of false acceptance, while NAA and NIA are the numbers of the authorized person attempts and the numbers of impostor person attempts respectively (Zhang, 2000). Furthermore, low FRR and low FAR is the main objective in order to achieve both high usability and high security of the system.

\section{Data set and experimental results}

The Chinese Academy of Sciences-Institute of Automation (CASIA) eye image database is used in the experiment. To evaluate the effectiveness of the proposed system, a database of 42 grayscale eye images (7 eyes with 6 different images for each eye) was employed. About 30 grayscale eye images with 5 unique eyes are considered as authorized users and the others are impostors as shown in Fig. 8.

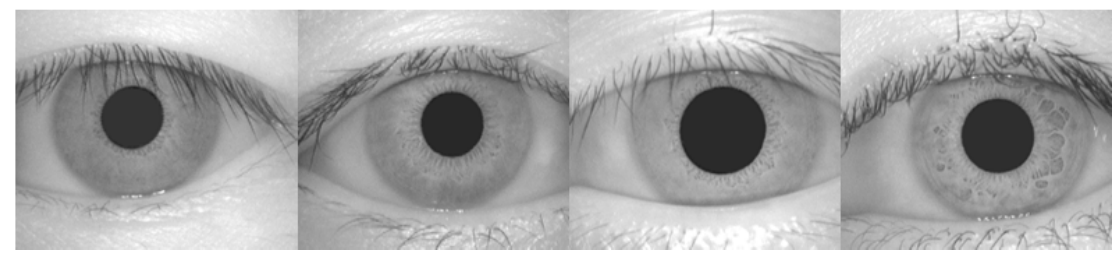

Fig. 8. Example of the iris images for different users.

For each eye, 6 eye images were captured in two different sessions with one month interval between sessions (three samples are collected in the first session and others three in second sessions) using specialized digital optics developed by the National Laboratory of Pattern Recognition, China. Infra-red lighting was used in acquiring the images, hence features in the iris region are highly visible and there is good contrast between pupil, iris and sclera regions.

As previously discussed, the performance of biometric systems is usually described by two error rates: FRR and FAR. Hence, the effectiveness of the proposed system in testing (operational) phase is evaluated based upon FRR and FAR values. The FAR is calculated 
based on the close set and open set. In the close set, the typing biometric of an authorized person uses other authorized person identity. On other hand, the open set is referred as typing biometric of the impostors use authorized person.

The obtained feature vector of iris code comprises matrix of $20 \times 480$. This feature vector consists of bits 0 and 1 . It was observed in all the experiments conducted that the feature vector size which containing high dimensionality often contributed to high FRR and FAR values with long processing time.

To overcome this problem, feature vector size is transformed to one-dimension vector which reduces to $1 \times 480$ by using averaging techniques (each segment is divided by 20) contains the average value. Experimental results are subsequently discussed in the following section.

\subsection{SVM-based iris code model}

SVMs are classifiers which have demonstrated high capability in solving variety of problems that include the object recognition problems. Experimental results of training and testing based on iris code using SVMs are discussed.

In developing user models based on iris code, a SVM with polynomial kernel function of order 8 is used. Each authorized user has its own SVM-based model characterized by a set of support vectors. By using quadratic programming in the MATLAB environment, appropriate support vectors are determined. The penalty term $C$ of 1015 is used to anticipate misclassified data. Table 2 shows the training performance when the SVM is employed to develop user's models based on their iris code.

\begin{tabular}{|c|c|c|}
\hline Authorized User & Training Time(sec) & $\begin{array}{c}\text { Classification } \\
\text { Result }(\%)\end{array}$ \\
\hline User 1 & 0.0781 & 100 \\
\hline User 2 & 0.0781 & 100 \\
\hline User 3 & 0.0625 & 100 \\
\hline User 4 & 0.1094 & 100 \\
\hline User 5 & 0.0781 & 100 \\
\hline Average & 0.0812 & 100 \\
\hline
\end{tabular}

Table 2. Training performances of iris code.

These results indicate that all of the SVM-based user models give perfect classifications as there are no errors in recognizing all the users. Besides, all of the SVM- based models can be trained in a very short time of about 0.1 second. Consequently, the SVM model should be further investigated and adopted for use in the proposed system.

A series of experiments is conducted using the testing data which have not been used during the training phase. Table 3 shows the testing performances of the SVM-based authorized user models.

The SVM-based authentication gives very good results for FAR of close set and open set conditions. This implies that the proposed system is well protected from attacking by impostors. In contrast, the FRR values are very high percentage with an average value of about $19.80 \%$. Hence, the system seems to have poor usability. Experimental results show that the first, second and fourth users produce maximum FRR values of about 33\%. Further study and improvement should be done before incorporating this model to the system. 


\begin{tabular}{|c|c|c|c|}
\hline Authorized User & FRR (\%) & $\begin{array}{c}\text { FAR (\%) } \\
\text { Close Set }\end{array}$ & $\begin{array}{c}\text { FAR (\%) } \\
\text { Open Set }\end{array}$ \\
\hline User 1 & 33 & 0 & 0 \\
\hline User 2 & 33 & 0 & 0 \\
\hline User 3 & 0 & 0 & 0 \\
\hline User 4 & 33 & 0 & 0 \\
\hline User 5 & 0 & 0 & 0 \\
\hline Average & 19.80 & 0 & 0 \\
\hline
\end{tabular}

Table 3. Testing performances of iris code.

\section{Conclusion}

This chapter has presented an iris recognition system, which was tested using database of grayscale eye images in order to verify the authorized user of iris recognition technology. Firstly segmenting method was used to localize the iris region from the eye image. Next, the localized iris image was normalized to eliminate dimensional inconsistencies between iris regions using Daugman's rubber sheet model. Finally features of the iris region were encoded by convolving the normalized iris region with 1D Log-Gabor filters and phase quantizing the output in order to produce a bit-wise biometric template.

The Support Vector Machine was adopted as classifier in order to develop the user model based on his/her iris code data. Experimental study using CASIA database is carried out to evaluate the effectiveness of the proposed system. Based on obtained results, SVM classifier produces excellent FAR value for both open and close set condition. Thus, the proposed system seems in a good level of security. However, further study has to be done to improve level of usability by reduce the value of FRR.

\section{Acknowledgment}

Portion of the research in this project work use CASIA iris image database (CASIA, 2003) collected by Institute of Automation, Chinese Academy of Science.

\section{References}

Burges, C. J. C. (1998). A tutorial on support vector machines for pattern recognition. Data Mining and Knowledge Discovery, 2 (2), 121-167.

Chinese Academy of Sciences-Institute of Automation (2003). Database of greyscale eyes images. Version 1.0.

http:/ / www.sinobiometrics.com

Daugman, J. (2002). How iris recognition works. Paper presented at the International Conference on Image Processing, Vol. 1, pp. 33-36.

Daugman, J. (2003). The importance of being random: statistical principles of iris recognition. Pattern Recognition, 36 (2), 279-291.

Field, D. (1987). Relations between the statistics of natural images and the response properties of cortical cells. Journal of the Optical Society of America, pp. 42-72. 
Hasimah, A. (2008). Design and development of integrated biometic system for high level security.(Master Thesis). Kulliyyah of Engineering. International Islamic University Malaysia.

Lim, S., Lee, K., Byeon, O., \& Kim, T. (2001). Efficient iris recognition through improvement of feature vector and classifier. ETRI Journal, 23 (2), 61-70.

Masek, L. (2003). Recognition of human iris patterns for biometric identification [B]. School of Computer Science and Software Engineering. University of Western Australia.

Public source of the MATLAB source code for iris recognition software is available: http://www.csse.uwa.edu.au/ masek101/

Shiavi, R. (1991). Introduction to applied statistical signal analysis. Homewood III.

Widles, R., Asmuth, J., Green, G., Hsu, S., Kolezynski, R., Matey, J.,\& McBride, S. (1996). A machine-vision system for iris recognition. Machine Vision and Application, Vol. 9, pp. 1-8.

Wildes, R. (1999). Iris recognition: an emerging biometric technology. IEEE, 85 (9).

Zhang, D. (2000). Automated biometrics technologies and system. Kluwer Academic Publishers.

Zhu, Y., Tan, T., \& Wang, Y. (2000). Biometric personal identification based on iris patterns. Paper presented at the $15^{\text {th }}$ International Conference on Pattern Recognition, Spain, Vol. 2, pp. 801-804. 


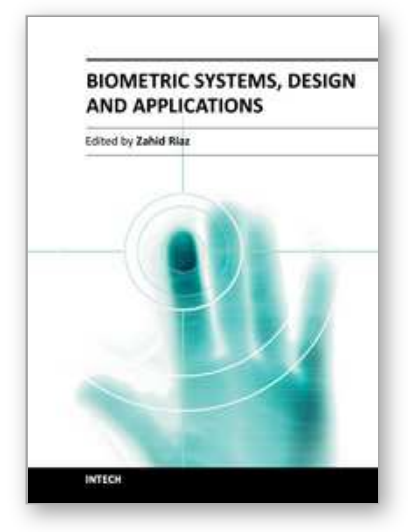

\author{
Biometric Systems, Design and Applications \\ Edited by Mr Zahid Riaz
}

ISBN 978-953-307-542-6

Hard cover, 262 pages

Publisher InTech

Published online 21, October, 2011

Published in print edition October, 2011

Biometric authentication has been widely used for access control and security systems over the past few years. The purpose of this book is to provide the readers with life cycle of different biometric authentication systems from their design and development to qualification and final application. The major systems discussed in this book include fingerprint identification, face recognition, iris segmentation and classification, signature verification and other miscellaneous systems which describe management policies of biometrics, reliability measures, pressure based typing and signature verification, bio-chemical systems and behavioral characteristics. In summary, this book provides the students and the researchers with different approaches to develop biometric authentication systems and at the same time includes state-of-the-art approaches in their design and development. The approaches have been thoroughly tested on standard databases and in real world applications.

\title{
How to reference
}

In order to correctly reference this scholarly work, feel free to copy and paste the following:

Hasimah Ali and Momoh J. E. Salami (2011). Iris Recognition System Using Support Vector Machines, Biometric Systems, Design and Applications, Mr Zahid Riaz (Ed.), ISBN: 978-953-307-542-6, InTech, Available from: http://www.intechopen.com/books/biometric-systems-design-and-applications/iris-recognition-systemusing-support-vector-machines

\section{INTECH}

open science | open minds

\author{
InTech Europe \\ University Campus STeP Ri \\ Slavka Krautzeka 83/A \\ 51000 Rijeka, Croatia \\ Phone: +385 (51) 770447 \\ Fax: +385 (51) 686166 \\ www.intechopen.com
}

\author{
InTech China \\ Unit 405, Office Block, Hotel Equatorial Shanghai \\ No.65, Yan An Road (West), Shanghai, 200040, China \\ 中国上海市延安西路65号上海国际贵都大饭店办公楼 405 单元 \\ Phone: +86-21-62489820 \\ Fax: $+86-21-62489821$
}


(C) 2011 The Author(s). Licensee IntechOpen. This is an open access article distributed under the terms of the Creative Commons Attribution 3.0 License, which permits unrestricted use, distribution, and reproduction in any medium, provided the original work is properly cited. 